\title{
Devnagari numeral recognition by combining decision of multiple connectionist classifiers
}

\author{
REENA BAJAJ $^{1}$, LIPIKA DEY $^{2}$ and SANTANU CHAUDHURY ${ }^{1 *}$ \\ ${ }^{1}$ Department of Electrical Engineering, Indian Institute of Technology, \\ New Delhi 110016, India \\ ${ }^{2}$ Department of Mathematics, Indian Institute of Technology, New Delhi 110016, \\ India \\ e-mail: santanuc@ee.iitd.ernet.in
}

\begin{abstract}
This paper is concerned with recognition of handwritten Devnagari numerals. The basic objective of the present work is to provide an efficient and reliable technique for recognition of handwritten numerals. Three different types of features have been used for classification of numerals. A multi-classifier connectionist architecture has been proposed for increasing reliability of the recognition results. Experimental results show that the technique is effective and reliable.
\end{abstract}

Keywords. Numeral recognition; meta-pi network; style category; classifier combination.

\section{Introduction}

Recognition of handwritten numerals is important because of its applicability to a number of problems, like postal code recognition and information extraction from fields of different forms. In the Indian context, there exists a need for development and/or evaluation of the existing techniques for recognition of numerals written in Indian scripts. Generic techniques cannot, in general, tackle problems associated with script specific peculiarities. In this paper, we present a neural network-based architecture for recognition of handwritten numerals. Although the architecture is generic, it has been found to be useful for recognition of handwritten Devnagari numerals.

Many schemes for digit classification have been reported in literature. They mostly differ in feature extraction schemes and classification strategies (Govindan \& Shivaprasad 1990; Trier et al 1996). Features used for recognition tasks include topological features, mathematical moments etc. Classification schemes applied include nearest neighbour schemes and feedforward networks. In order to make their systems robust against variations in numeral shapes, researchers have also used deformable models, multiple algorithms and learning. A recent survey of the techniques is provided by Amin (1997) and Plamondon \& Srihari (2000). Lam $\&$ Suen (1986) have used a fast structural classifier and a relaxation-based scheme which uses

*For correspondence 
deformation for matching. A knowledge-based system using multiple experts has been used by Mai \& Suen (1990). Kimura \& Sridhar (1991) developed a statistical classification technique that utilised profiles and histograms of the direction vectors derived from the contours. Chen \& Lieh (1990) proposed a two-layer random graph-based scheme which used components and strokes as primitives. Jain \& Zongkar (1997) have proposed a recognition scheme using deformable templates. LeCun et al (1989). suggested a novel backpropagation-based neural network architecture for handwritten zip code recognition. Knerr et al (1992) suggested the use of neural network classifiers with single-layer training for recognition of handwritten numerals. Wang \& Jean (1993) suggested use of neural networks for resolving confusion between similar looking characters.

Among studies on Indian scripts, notable work has been done on recognition of printed Devnagari characters by Sinha and others (Sinha \& Mahabala 1979; Bansal \& Sinha 2001). They also suggested contextual post-processing for Devnagari character recognition and text understanding. For handwritten Bengali character recognition, Dutta \& Chaudhury (1993) presented a curvature feature based approach. Chaudhuri \& Pal (1998) presented a complete Bangla OCR system.

In this paper, we present a new approach for recognition of handwritten Devnagari numerals using multiple neural classifiers. In this work we have tried to exploit information about stylistic variations, similarity between numerals and style invariant features. A combination of these three different types of information (not simply different types of features) for the purpose of recognition has not been attempted before. We have proposed a novel neural network-based architecture for integration of multiple sources of information. Experiments have shown that this combination scheme has the power to eliminate confusion and to provide reliable recognition performance. In the next subsection, we provide an overview of the scheme, highlighting the advantages of the proposed approach.

\subsection{Overview of the scheme}

In this paper, we propose a recognition architecture using connectionist networks as basic building blocks. Connectionist networks have been used because of their inherent capability to learn and generalise. In the present work the numerals have been represented using two types of features. The first type of features provide coarse shape description of the numerals and are relatively insensitive to minor changes in character shapes. But these features are not expected to remain unchanged for different writing styles (typical of different individuals). The second class of features try to provide qualitative descriptions of the characters. These descriptions encode intrinsic properties of the characters which are expected to be invariant across writing styles and fonts.

The initial stage of the proposed architecture consists of connectionist modules for style-based categorisation of the input. Since style groups are characteristic of each character, for each character there exists a style categorisation module which acquires knowledge about style categories of the corresponding character through unsupervised learning. Output of this stage would indicate similarity of an unknown input with style categories of the different characters (including the correct one). An interesting feature of this stage is that the classifiers are not forced to classify distinct looking samples of one character into one monolithic class. The classifiers can self-organise themselves to categorise them into distinct style categories. These categories refer to individual characters thereby minimising learning interference because of similar samples of other characters. So the first step in recognising an unknown sample involves determining how similar the character is with writing styles of 
different characters. Use of style categories in the recognition process is a novel innovation proposed in this scheme.

To identify the correct classification label, the second layer of the recognition architecture consists of a feed-forward network trained through a supervised learning process to distinguish the correct character from others. For each character there is one feed-forward recognition net with one output node. The network receives input from each style categorisation module. The feed-forward net can therefore integrate similarity information to arrive at the correct recognition decision. For each character, we have considered distinct networks because the network needs to learn character specific similarity characteristics to perform correct classification. Samples of 1 may be at times similar to 7 - the classifier must learn this information and at the same time it should learn to reject samples of 7 which are distinct from 1. Implementation of these conflicting requirements using a single classifier (for all numerals) would have made the learning process complex and slow. Another advantage of this modular structure is extendability. Addition of a new character to the set leads to the addition of a fixed number of additional links to each network and one additional classification module. In other words, complexity of the network does not show exponential growth. Use of a supervised learning scheme for integration of style-dependent similarity information is a new contribution of this work. Given an unknown sample, a network-module will indicate the degree to which the sample is similar to the particular character represented by this classifier. Provision for obtaining alternative classification for an unknown character is another advantage of this architecture. Therefore, first two layers of the recognition system provide the facility for using style-dependent information for classification of a character.

The second type of features used here provides qualitative description of the characters in terms of the presence or absense of specific types of primitives in the numeral. A self-organising learning system has been used to identify the primitive types for the given sample set. Using the information about presence and absense of primitives, a simple MLP-based classifier performs the categorisation of the numerals. The output of this classifier is fed into the third stage of the recognition architecture so that style-specific and style-invariant information can be combined for the recognition problem.

The third layer in the recognition network is the integration layer. This layer can integrate the output of more than one classifier to resolve ambiguities to provide the final classification result. Here also we have used a simple feed-forward net which has been trained through a supervised learning scheme to remove ambiguities. Ambiguities can be resolved on the basis of an assesment of the performance of the classifiers for the known sample sets. The overall integration architecture which enables fusion of style-specific and style-invariant information for character recognition is an important contribution of this paper.

\section{Feature extraction}

In this work we have considered the numerals to be unconstrained, isolated, and clearly discriminated against the background. Some sample numerals are presented in figure 1.

The scanned image of the character is preprocessed to remove noise. A median filtering-based approach is followed for filtering. A similarity transformation invariant representation for each numeral is obtained by using the principal component analysis as suggested by Lee \& Pan (1992). This representation is referred to as the normalised character image. These numeral images are also thinned for the purpose of descriptive feature extraction using the SPTA algorithm (Naccache \& Singhal 1984). Results of preprocessing are presented in figure 2. 


$$
\begin{array}{lllllll}
0 & 1 & 2 & 2 & 8 & y & \xi \\
0 & 1 & 2 & 3 & 8 & y & \xi \\
0 & 1 & 2 & 3 & 8 & y & \xi \\
0 & 1 & 2 & 2 & 8 & y & \xi \\
0 & 1 & 2 & 3 & 8 & y & \xi
\end{array}
$$

Figure 1. Sample data set.

For the purpose of classification each numeral is represented using three types of features. Features used in this approach are as listed below.

- Density features.

- Moment features of right, left, upper and lower profile curves.

- Descriptive component features.

\subsection{Density feature extraction}

Shape of individual numerals can be characterised by considering distribution of dark pixels over image regions. Relative densities of the pixels over these regions characterise the shape of the character at reduced resolution. Consequently, minor variations in individual character<smiles>[Mg]=C1CCCCC1</smiles>

(a) (b) (c)
)
$\mathbf{F}$ Figure 2. Results of preprocessing: (a) Raw sample, (d) (b) blurred images, (c) thinned image, (d) normalised image. 


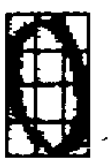

\begin{tabular}{|l|l|l|}
\hline 0.615 & 0.419 & 0.482 \\
\hline 0.582 & 0.010 & 0.000 \\
\hline 0.600 & 0.548 & 0.000 \\
\hline 0.332 & 0.366 & 0.366 \\
\hline
\end{tabular}

\begin{tabular}{|c|c|c|}
\hline 0.495 & 0.152 & 0.571 \\
\hline 0.952 & 0.036 & 0.610 \\
\hline 0.038 & 0.836 & 0.319 \\
\hline 0.343 & 0.000 & 0.000 \\
\hline
\end{tabular}

\begin{tabular}{|l|l|l|}
\hline 0.681 & 0.438 & 0.243 \\
\hline 0.568 & 0.009 & 0.276 \\
\hline 0.746 & 0.924 & 0.013 \\
\hline 0.438 & 0.000 & 0.000 \\
\hline
\end{tabular}

Figure 3. Results of density feature extraction (table shows the density feature values).

instances are masked out. But at the same time, stroke width, a determining factor for the density features, is a style-dependent phenomenon. Therefore, density features can be considered to encode style characteristics without being affected by variations in minute details of similarly formed characters.

To extract local density feature, a $3 \times 4$ grid is overlaid on the normalised numeral. The grid boundaries are co-incident with the bounding box of the numeral. Density feature for a single element of grid is calculated as:

$$
d(m, n)=\frac{\text { number of dark points in the element denoted by } m, n}{\text { total number of points in this grid element }}
$$

Density features for some of the numerals are shown in figure 3.

\subsection{Profile curve-based approach}

Connecting extremal points found along four directions of the normalised character image, we can obtain four profile curves for the character shapes. These profile curves also encode shape information of the numerals. Normalized moments of these profile curves are computed in the following way:

Let $X^{\prime}$ and $Y^{\prime}$ represent the $x$ and $y$ coordinates of the centre of mass. Moment of $r$ th order is calculated using the formula:

$$
\mu_{r}=\sum\left(x-x^{\prime}\right)^{r} \operatorname{pro}(x) \mathrm{d} x,
$$

where $\mu_{r}$ is the moment of order $r$ and $\operatorname{pro}(x)$ is the profile value (as profile is a onedimensional curve). 
Using moments of order 2, 3 and 4, we try to obtain measures of Kurtosis and skewness for the numeral. In this way we have obtained three features of each profile (total 12). The features are described as follows:

(1) Measure of Kurtosis, which is a measure of flatness of the distribution.

$$
K V=\mu_{4} /\left(\mu_{2}\right)^{2} .
$$

(2) Measure of skewness, which is a measure of the assymmetry of the distribution.

$$
S V=\mu_{3} /\left(\mu_{2}\right)^{1.5} .
$$

(3) Measures of normalized skewness and Kurtosis, which relate the symmetry to flatness of the distribution.

$$
N V=\mu_{3} /\left(\mu_{4}\right)^{0.75}
$$

The moment measures considered here provide gross characterisation of the shape of the profile curves. Consequently, the measures are relatively unaffected by minor variations in the numeral shape. But distinct differences in the shape of the character because of variations in writing style or intrinsic nature of the shape are adequately captured by these features. Profile curves and moment feature of a numeral are shown in figure 4.

\subsection{Descriptive features}

In figure 1, a sample set is presented. With reference to the sample set we can observe that numerals are composed of strokes of different types. A description of the character can be constructed in terms of these constituent strokes. Constituent components of a character can be identified by considering the junction points of the numerals where multiple branches meet. These meeting points (if any) are universally present in all samples of the same numeral. Distinct branches meeting at these points can be considered as constituent primitives. When junction points are missing we can consider the numeral to be a simple shape composed of a single primitive. Therefore, a style invariant representation of the characters can be obtained in terms of the primitive types present in a character.

From a thinned image of a numeral, junctions and individual segments are extracted and appropriately represented for the recognition task. These segments can be characterised by their lengths, average directivity, average directivity strength and the average directivity change. Though the length of individual segment can vary from one sample to other, the size is in general proportional to the size of the numeral. Similarly, the directivity of individual dark pixels may vary quite a lot but in terms of average values for individual segments, these are more or less constant. The feature extraction process consists of the following steps: identification of junctions, tracking and identification of segments, generation of feature vectors for segments and feature vector construction for each character.

2.3a Generation of feature vectors: The feature vector for the segments consists of the following feature fields:

(1) Normalized length of the segment;

(2) average directivity of the segment;

(3) average directivity strength of the segment;

(4) average directivity change of the segment. 


\begin{tabular}{|l|r|r|r|}
\hline & $\mathrm{KV}$ & $\mathrm{SV}$ & $\mathrm{NV}$ \\
\hline left & $\mathbf{0 . 0 1 7}$ & 0.118 & 2.536 \\
\hline right & $\mathbf{0 . 0 0 7}$ & -0.037 & -1.601 \\
\hline top & 0.012 & 0.107 & 2.901 \\
\hline bottom & 0.008 & 0.087 & 3.269 \\
\hline
\end{tabular}<smiles>[C]1CC1</smiles>

top

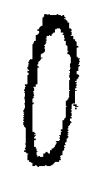

$r$

top

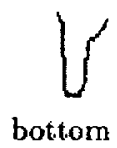

botiom

\begin{tabular}{|l|r|r|r|}
\hline & $\mathrm{KV}$ & $\mathrm{SV}$ & $\mathrm{NV}$ \\
\hline left & 0.018 & 0.131 & 2.642 \\
\hline right & 0.034 & 0.162 & 2.035 \\
\hline top & 0.163 & 0.126 & 2.769 \\
\hline bottom & 0.299 & 0.170 & 2.361 \\
\hline
\end{tabular}

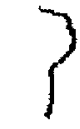

right

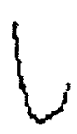

bottom

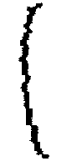

left

Figure 4. Profile features (table shows the moment features).

Values of these feature fields are computed following the technique proposed by Suganuma (1991):

(1) A count of the number pixels in the thinned segment is obtained during segment tracing. This is normalized with respect to the area of the bounding box of the character. This feature, in a way, is a measure of the space-filling property of the corresponding segment.

(2) Average directivity of the segment is calculated as follows.

$$
\sum_{i}\left\{\operatorname{erstr}_{i} * \operatorname{erdir}_{i}\right\} / \sum_{i} \text { erstr }_{i}
$$

where $\operatorname{erdir}_{i}$ and erstr $_{i}$ are respectively the directivity and directivity strength of an element in a segment. These parameters are determined by considering a $3 \times 3$ neighbourhood around each pixel.

(3) Average directivity strength is defined as:

$$
\sum_{i} \frac{\text { erstri }_{i}}{\text { segment length }}
$$

The segment length is measured in number of pixels. 

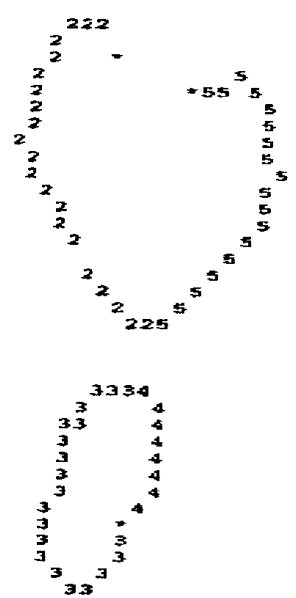

(a)

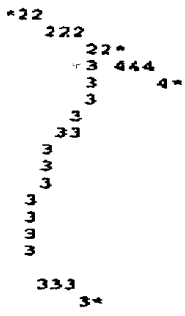

(a)

\begin{tabular}{|c|c|c|c|c|}
\hline$n-$ sgmt & $n$ - elmt & dir & stdir & chdir \\
\hline 1 & 0.021 & 0.754 & 0.192 & 0.031 \\
\hline 2 & 0.073 & 0.812 & 0.111 & 0.023 \\
\hline 3 & 0.017 & 0.789 & 0.505 & 0.008 \\
\hline
\end{tabular}

(b)

\begin{tabular}{|c|c|c|c|c|}
\hline$n-$ sgmt & $n-$ elmt & dir & stdir & chdir \\
\hline 1 & 0.059 & 0.819 & 0.218 & 0.162 \\
\hline 2 & 0.043 & 0.769 & 0.253 & 0.040 \\
\hline
\end{tabular}

(b)

Figure 5. Descriptive features: (a) segments identified, (b) corresponding descriptive features.

(4) Directivity change which is obtained by getting the difference of directivity of two immediate neighbours of each element of the segment. The average is taken to get the normalized results.

Segments of a numeral and the corresponding feature vectors of the numeral has been shown in figure 5 .

2.3b Character representation: Using the feature vectors generated for each segment, types of the primitives involved in composition of the characters have been identified through an unsupervised clustering scheme. Clustering is performed using Kohonen's self-organising feature maps. We have adopted Kohonen's algorithm because it has the ability to identify categories exploiting the distribution of feature vectors (like any other unsupervised learning scheme) and at the same time imposes an order on these categories based upon similarity between them. A modified Kohonen's learning algorithm (Sabourin \& Mitiche 1993) which adaptively finds the number of distinct categories has been used here.

A Kohonen network provides a topology-preserving map from the space of inputs to the space of outputs. The map preserves the neighbourhood relation defined for the output space. During the learning phase, models or prototypes are learned by adjusting the weights of the output neurons and its neighbours. For every training example, we find the closest output cell (i.e. the output cell with associated weight vector having minimum Euclidean distance with the input pattern) and move the weights of the output unit and its neighbours closer to the input pattern. Finally, the models, and the distance measure used to generate the models, induce a partition, $S_{i}$, of the pattern space, specified as:

$$
S_{i}=\left\{x_{j} / d\left(x_{j}, y_{i}\right)<d\left(x_{j}, y_{k}\right) \forall k \neq i\right\} .
$$


Thus, to each input node $i$ we associate a partition $S_{i}$. We state that region $S_{i}$ is homogeneous, if the variance of the partition is less than a threshold. In the modified algorithm we first generate a Kohonen's net with a fixed number of output units (say, $N$ ). If the homogeneity condition is violated for a partition induced by a node then we generate a new Kohonen net with at least $(N+1)$ units. This process is repeated till the homogeneity condition is achieved for all the partitions induced by the net.

Now, a character can be represented using a binary feature vector which would indicate whether a particular type of primitive is present in the character or not. In fact, in the present approach a generalised representation scheme is used. The feature vector is constructed by considering normalised response of the winning Kohonen unit for individual segments. Each output unit in the Kohonen net uses a Gaussian function for output generation. The mean of the Gaussian function is the same as that of the weight linked with the unit and variance is defined by the final partition obtained during training.

\section{Numeral classification}

A neural network-based classification scheme is designed for this task. The numerals are represented by feature vectors of three types. Three different neural classifiers have been used for classification of these numerals. Finally, the outputs of the three classifiers are combined using a connectionist scheme. The network architecture has the following components.

(1) Kohonen self-organising maps at the lowest layer;

(2) A single-layer super-structure laid on Kohonen nets;

(3) Multi-layer-perception (MLP)-based classifier for the segment features;

(4) Meta-pi combining net for integration.

A 3-layer MLP is used for implementing the classifier for segment-based features. The net was trained with the back-propagation algorithm. Dimensionality of the input vector is the same as that of the number of segment types identified in the training set.

A diagrammatic representation of the complete architecture is presented in figure 6. Given an unknown input numeral pattern, we extract density features, profile features and segment type-based features. Using Kohonen net-based style specific classifiers the input pattern is classified into style categories. The combination layer combines the output of style categories corresponding to different numerals and provides inputs for the meta-pi integration network. For the final classification we adopt a winner-take-all strategy. The global output unit having maximum activation provides the identity of the numeral.

\subsection{Kohonen net-based modules}

The Kohonen net is used at the lower layer to identify distinct stylistic variations in a single character. An unsupervised learning strategy like the Kohonen net is the only applicable scheme to learn style categories corresponding to a particular character because of the following reasons.

(1) Normally it is not possible to know a priori the style categories for a given sample set.

(2) A priori style labellings of the samples are also not available.

Style clusters are expected to emerge through the Kohonen self-organising learning scheme. With this objective, for each numeral we train individual Kohonen nets with both density 




Figure 6. Representation of the recognition architecture.

and profile feature vectors. For learning we use the same multipass unsupervised learning algorithm as described in the previous section.

The output of the winning unit of the Kohonen module indicates the degree of similarity (defined by the Euclidean distance measure used for learning) of the input with the style prototype learnt because of the Gaussian response function associated with each output unit of the Kohonen module. The partitions induced by the prototype vectors representing style categories of each numeral may overlap in space. This property is exploited by the combination scheme.

\subsection{Combining output of the Kohonen modules}

The activation of the output nodes of the individual Kohonen modules provides a measure of the degree of similarity of the input sample with different character styles. Since style categories overlap, more than one Kohonen module can provide significant outputs for an input sample. These outputs are combined in order to obtain a classification result on the basis of similarity between different characters. A linear combination rule for the similarity scores 
has been used. Weights associated with the different modules are determined on the basis of the relative significance and relevance of the individual modules. Consider an example. For correct classification of an input sample as "1" (English) weight associated with the output of Kohonen module for "1" would be maximum, followed by that for "7"; weight associated with output of " 5 " may be much less.

For combining outputs, a single-layer feed-forward net is used. Each element in the net is an ADALINE (adaptive linear element). Bias input is set to zero. For each numeral, there is one output unit. The winning unit of of each Kohonen module provides the input for the adaline units. Activation $\left(A_{i}\right)$ of the $i$ th unit is given by $A=\sum_{j} w_{j}^{i} O_{j}$ where $O_{j}$ is the output of the winning unit of the $j$ th Kohonen module and $w_{j}^{i}$ is the weight of the link connecting the $j$ th module with the $i$ th adaline unit. Using the Widrow learning rule, based upon LMS, weights are learned. These weights reflect the relative significance of each module's contribution. These network is trained using the sample set which includes newer samples in addition to the samples used for training individual Kohonen modules. This super- structure used for combining the outputs of the Kohonen module has distinct resemblance with a radial basis function network. However, the structure of the intermediate layer is different. Instead of all RBF nodes in the intermediate layer, only the winning node in the Kohonen module of each numeral provides activation for the output layer.

\subsection{Classifier combination}

The classifiers used in this approach are based upon different representations of the input pattern. These representations, since they encode different types of property - style-dependent and style-invariant, cannot be combined into a single monolithic feature vector. Individual classifiers dealing with these representations output the class labels depending on the features used. These class labels has been combined using a meta-pi network (Hampshire \& Waibel (1992) because it can devise a combination scheme on the basis of consistency and accuracy of the individual classifiers. A meta-pi network constitutes a Bayesian MAP classifier (Hampshire \& Waibel 1992). Each output unit of the meta-pi net modulates output of individual classifiers. The network is called the meta-pi network owing to the multiplicative function that its output units perform. This function serves to combine the outputs of sub-networks (or "modules"), independently trained to classify numerals based on different features. Hence, there are three output nodes in the present meta-pi net. The network has been trained with the samples used for training the individual nets along with newer examples. Through the training process the meta-pi net learns to choose any one of the valid classifier outputs or a combination of these valid outputs to produce the correct global output.

Meta-pi net is an ordinary 3-layer backpropagation net with three output units. The global output of the net for the numeral $n$ is given by:

$$
O_{n}=\frac{1}{\mu_{n}} \sum_{k} \rho_{k, n} M_{k},
$$

where,

$$
\begin{aligned}
\mu_{n} & \equiv \sum_{k} M_{k n}, \\
\mu_{n} & >0, \\
0 & \leq M_{k n} \leq 1 \quad \forall k, \forall n,
\end{aligned}
$$


Table 1. Results of Kohonen module (for numeral 5) for the density feature.

\begin{tabular}{llllll}
\hline \multirow{2}{*}{ Person } & \multicolumn{5}{c}{$\begin{array}{c}\text { No. of samples in } \\
\text { style categories }\end{array}$} \\
\cline { 2 - 5 } & 1 & 2 & 3 & 4 & 5 \\
\hline 1 & 5 & & & & 1 \\
2 & & & & 1 & 5 \\
3 & 6 & & & & \\
4 & & & 4 & & 2 \\
5 & & 6 & & & \\
6 & 1 & & 1 & 4 & \\
7 & & 4 & & 1 & 1 \\
8 & & & 3 & & 3 \\
\hline
\end{tabular}

where $\rho_{k, n}$ is the output of the $k$ th classifier corresponding to the $n$th numeral and $M_{k}$ is the continuous valued output from the $k$ th output unit of the meta-pi net. The learning law is same as that given by Naccache \& Singhal (1984). Depending on the strength of the global output we can rank the recognition hypotheses. Consequently, we can have alternative classifications for an unknown input.

The meta-pi net receives, as input, an image of the character at reduced resolution. The $4 \times 3$ grid used for density feature extraction is used for constructing a binary input feature vector. If the density value at a grid is found to be more than 0.3 then the corresponding component in the feature vector is set to 1 , otherwise it is set to 0 .

\section{Results and discussion}

A prototype recognition system was implemented using $C$ on Sun's Spark System. For experimentation, samples were collected from a number of different individuals. Examples of some of the data used are shown in figure 1.

Four samples of each numeral from eight different persons were used in the training of the neural net. For every feature set, ten SMR modules were trained independently for each numeral. Three to six style classes were obtained depending on the type of numeral. For descriptive features twelve types of segments were identified. Twenty additional samples were used for training the feed-forward superstructure of the Kohonen modules and the integration network.

The capability of the Kohonen net in identifying style categories becomes clear from the results in table 1 . The Kohonen module for numeral five trained with density features was used for categorising samples of the numeral five written by different individuals. It is expected that a person writes a numeral in one or two distinct ways. Results of the categorisation experiment vindicated this fact. Numerals written by an individual have been categorised into atmost two style categories. Although results of only one module have been presented here, similar capabilities are observed for other modules as well. It is also found that these style modules have rejection capability (for other numerals) of around seventy percent on average.

The classification scheme was tested with 2460 different samples drawn from different individuals. Results are presented in table 2 . 
Table 2. Classification results (in percentages).

\begin{tabular}{lcc}
\hline Classifier & Correct classification & Wrong classfication \\
\hline Density & 39.57 & 31.5 \\
Segment & 50.2 & 22.5 \\
Moment & 49.36 & 20.42 \\
Moment and density & 63.4 & 31.7 \\
Density and segment & 84.04 & 14.5 \\
Moment and segment & 75.5 & 20.42 \\
All three & 89.68 & 8.17 \\
\hline
\end{tabular}

For an individual classifier the class inferred corresponds to the nodes on the output layer having maximal activation, provided the activation is greater than 0.2 . In this case, obviously the integration network does not have a role to play. From the results in the table, it is clear that there is substantial reduction of error rate when the outputs of the classifiers are combined using the meta-pi net. Non-availability of benchmark data prevents us from experimenting with larger data set.

\section{Conclusions}

This paper is concerned with the problem of recognition of unconstrained, isolated, handwritten Devnagari numerals. The novel feature of this work is the approach followed for identification and integration of style specific information in the recognition scheme. Use of multiple classifiers using the meta-pi network is another significant feature of this work. A complete hierarchical recognition architecture has been suggested in this work. This architecture is applicable for any character recognition problem. Experimental results show that this approach is potentially powerful.

\section{References}

Amin A 1997 Off-line Arabic character recognition: Survey. Proc. 4th Int. Conf. on Document Analysis and Recognition, Munich (IEEE Press)

Bansal V, Sinha R M K 2001 A complete OCR for printed Hindi text in Devnagari script. Proc. 6th Int. Conf. on Document Analysis and Recognition, Washington (IEEE Press) (to appear)

Chaudhuri B B, Pal U 1998 A complete printed Bangla OCR system. Pattern Recogn. 31: 531-549

Chen L-H, Lieh J R 1990 Handwritten character recognition using a two layer random graph model by relaxation matching. Pattern Recogn. 23: 1189-1205

Dutta A K, Chaudhury S 1993 Bengali alpha-numeric character recognition using curvature features. Pattern Recogn. 26: 1757-1770

Govindan V K, Shivaprasad A P 1990 Character recognition - a review. Pattern Recogn. 23: 671-683

Hampshire J B II, Waibel A 1992 The meta-pi network: Building distributed knowledge representations for robust multisource pattern recognition. IEEE Trans. Pattern Anal. Machine Intell. PAMI-14: 751-769

Jain A K, Zongkar D 1997 Representation and recognition of handwritten digits using deformable templates. IEEE Trans. Pattern Anal. Machine Intell. PAMI-19: 1386-1391

Kimura F, Shridhar M 1991 Handwritten numerical recognition based on multiple algorithms. Pattern Recogn. 24: 969-983

Knerr S, Personnaz L, Dreyfus G 1992 Handwritten digit recognition by neural networks with singlelayer training. IEEE Trans. Neural Networks 3: 303-314 
Lam L, Suen C Y 1986 Structural classification and relaxation matching of totally unconstrained handwritten ZIP codes. Pattern Recogn. 19: 15-19

LeCun Y, Boser B, Denker J S, Henderson D, Howard R B, Hubbard W, Jackel L D 1989 Backpropagation applied to Handwritten zip code recognition. Neural Comput. 1: 541-551

Lee S, Pan J C 1992 Offline tracing and representation of signatures. IEEE Trans. Syst., Man Cybern. SMC-22: 755-771

Mai T, Suen C Y 1990 A generalised knowledge-based system for recognition of of unconstrained hand-written numerals. IEEE Trans. Syst., Man Cybern. SMC-20: 835-848

Naccache N J, Singhal R 1984 SPTA: A proposed algorithm for thinning binary pattern. IEEE Trans. Syst., Man Cybern. SMC-14: 409-418

Plamondon R, Srihari S N 2000 On-line and off-line handwriting recognition: comprehensive survey. IEEE Trans. Pattern Anal. Machine Intell. PAMI-22: 63-84

Sabourin M, Mitiche A 1993 Modeling and classification of shape using a Kohonen associative memory with selective multiresolution. Neural Networks 6: 275-283

Sinha R M K, Mahabala H 1979 Machine recognition of Devnagari script. IEEE Trans. Syst., Man Cybern. SMC-9: 435-449.

Suganuma Y 1991 Learning structures of visual patterns from single instances. Artif. Intell. 50: 1-36

Trier O D, Jain A K, Taxt T 1996 Feature extraction methods for character recognition - A survey. Pattern Recogn., 29: 641-662

Wang J, Jean J 1993 Resolving multifont character confusion with neural networks. Pattern Recogn. 26: $175-187$ 